\section{Redundant enhancers and causal variants in the TCF7L2 gene}

European Journal of Human Genetics (2014) 22, 1243-1246; doi:10.1038/ejhg.2014.17; published online 12 February 2014

The TCF7L2 gene is the locus with the strongest association with risk of type 2 diabetes (T2D) in different populations. ${ }^{1,2}$ The rs7903146 single-nucleotide polymorphism (SNP), located in intron 3 and the one with the strongest association to T2D risk, has been the most widely studied TCF7L2 variant. Current evidence suggests that rs7903146 may affect risk of T2D through its effects on enhancer activity $^{3,4}$ resulting in higher TCF7L2 gene expression. ${ }^{3-5}$ The rs7903146 SNP has been found located inside islet-selective open chromatin sites using either formaldehyde-assisted isolation of regulatory elements coupled with high throughput sequencing (FAIRE-seq), ${ }^{3}$ or histone $\mathrm{H} 3$ lysine methylation modifications as marks of regulatory elements. ${ }^{4}$ In addition, the T-allele of rs7903146 (ie, the allele associated with higher risk of T2D) has been found associated with higher TCF7L2 gene expression in human pancreatic islets, ${ }^{5}$ and in luciferase reporter assays in the mouse pancreatic MIN6 cell line, ${ }^{3,4}$ and the rat pancreatic 832/13 cell line. ${ }^{3}$ More recently Savic et $a l^{6,7}$ reported that the TCF7L2 gene harbors several highly conserved cis-regulatory elements that show both in vitro and in vivo enhancer activity. These enhancer elements are located in a 92-kb interval that harbors all the TCF7L2 SNPs, not just rs7903146, associated with T2D risk. The physical overlap between multiple enhancer elements and TCF7L2 variants suggests that this gene may carry more than one causal variant related to the development of T2D. The present letter discusses the implications of these recent findings and presents possible mechanisms in how genetic variants in the TCF7L2 gene may affect the development of T2D.

\section{Causal variants in TCF7L2}

Although the 92-kb enhancer-rich region in TCF7L2 harbors several SNPs that have been found associated with risk of T2D, it is commonly assumed the presence of a single causal variant in the region. ${ }^{8-10}$ However, a critical assessment of published results does not rule out the presence of an independent secondary signal. Helgason et $a l^{8}$ carried out haplotype analysis of SNPs, rs7903146 and rs12255372, and the microsatellite DG10S478 (ie, the three markers that were initially found with the strongest association with $\mathrm{T} 2 \mathrm{D}^{11}$ ) in Danish and Icelandic populations, and concluded that rs7903146 is the most likely causal variant. However, this same study also did single SNP analysis in West African groups, and although rs7903146 was indeed the marker with the strongest association with $\mathrm{T} 2 \mathrm{D}$ (odds ratio $(\mathrm{OR})=1.45,95 \%$ confidence interval $(\mathrm{CI})=1.19$ 1.77), the rs 12255372 showed a weaker but still significant association with $\mathrm{T} 2 \mathrm{D}(\mathrm{OR}=1.31,95 \% \mathrm{CI}=1.01-1.69)$. Given the nonexistent linkage disequilibrium (LD) between both SNPs in West African populations (YRI $r^{2}=0.003$ ), these results are consistent with the presence of at least two different causal variants in the TCF7L2 gene; a stronger signal tagged by rs7903146 and a weaker signal tagged by rs12255372. In contrast, the high LD between these two SNPs in European ancestry populations (CEU $\left.r^{2}=0.70\right)$ would mask the presence of the secondary signal. Palmer et al ${ }^{9}$ in a recent re-sequencing study of a small region of $4.3 \mathrm{~kb}$ region in the TCF7L2 gene in African Americans proposed rs7903146 as the causal T2D polymorphism. However, the re-sequenced region did not cover rs12255372 that is $50.5 \mathrm{~kb}$ away from rs7903146. It is worth noting that the same research group in an earlier study found both rs7903146 $(\mathrm{OR}=1.51,95 \% \mathrm{CI}=1.27-1.80)$ and $\mathrm{rs} 12255372(\mathrm{OR}=1.20,95 \%$ $\mathrm{CI}=1.00-1.43)$ being associated with risk of T2D in African Americans. ${ }^{12}$ Because these two SNPs are not correlated in African Americans (ASW $r^{2}=0.01$ ), these results also suggest the presence of at least two causal variants rather than a single causal variant. Maller et al $^{10}$ used Bayesian-based fine mapping in the Wellcome Trust Case Control Consortium (WTCCC) GWAS and estimated that $75 \%$ of the posterior weight of association in TCF7L2 rests on rs7903146. However, WTCCC data are inadequate to identify a secondary signal even if there is one present, given the high LD in the TCF7L2 gene in European populations. In point of fact, in the only three genes where the WTCCC study found evidence of additional independent signals for T2D, the secondary SNP was in low LD with the index SNP; FTO (index rs17817449 and secondary rs8063946, CEU $r^{2}=0.051$ ), CDKN2A (index rs12555274 and secondary rs10965250, CEU $r^{2}=0.067$ ), and CDKAL1 (index rs7756992 and secondary rs6456360, CEU $\left.r^{2}=0.006\right)$. Additional evidence of the relevance of rs12255372 independent from rs7903146 was reported by Parra et $a l^{13}$ who found that $\mathrm{rs} 12255372$ (OR $=1.78,95 \%$ $\mathrm{CI}=1.11-2.88)$ shows a stronger association with T2D in Hispanics relative to $\mathrm{rs} 7903146(\mathrm{OR}=1.39,95 \% \mathrm{CI}=0.89-2.17)$. Because the minor allele frequency (MAF) of rs12255372 was lower (11.6\%) than the MAF of rs7903146 (16.1\%) in the studied Hispanic population, the stronger association of rs12255372 cannot be attributed to higher statistical power compared with rs7903146. At last, a recent study found that rs12255372 but not rs7903146 interacts with dietary fat intake in relation to changes in body fat composition. ${ }^{14}$ In summary, these results do not rule out the role of rs12255372 as an independent secondary signal in the TCF7L2 gene.

\section{Alternative use of redundant enhancers}

In addition to the high LD that may be masking additional signals in TCF7L2 another possibility should be considered, namely that TCF7L2 enhancers may be redundant and they may show personto-person variation on their use. Although the actual number of enhancer elements in the TCF7L2 gene is still unclear, multiple enhancers that show overlapping activity in several cell lines and tissues are present in this region ${ }^{6,7}$ These observations raise the question whether some of those enhancer elements could be showing functional redundancy, as it has been shown for other genes with multiple enhancers. ${ }^{15,16}$ The Encyclopedia of DNA Elements (ENCODE) project has recently depicted a complex picture of chromatin interactions, where on average 3.9 enhancer elements physically interact with 1 transcription start site (TSS) and 2.5 TSSs physically interact with 1 enhancer element. ${ }^{17}$ The occurrence of a multitude of enhancer-TSS interactions raises the possibility that some of those interactions are interchangeable (ie, redundant) and that enhancer functional redundancy may be a general feature of gene 
expression regulation. Whether this is the case or all these enhancerTSS interactions are strictly necessary for normal gene expression is an open question that requires an answer.

Person-to-person variation in the use of redundant enhancers may add a layer of complexity to gene expression regulation and risk of disease. Recent evidence suggests the presence of interindividual variation in the use of alternative promoters ${ }^{18,19}$ and even usage differences between ethnic groups. ${ }^{20}$ The author recently proposed the hypothesis that person-to-person variation in the use of alternative regulatory elements may extend to both gene promoters and enhancers. ${ }^{21}$ This hypothesis predicts that, in presence of inter-individual variation in the use of redundant regulatory elements (eg, gene promoters and enhancers) and if functional SNPs are located inside those elements, the observed effects of these functional variants would be attenuated by a factor equal to the proportion of chromosomes in the population using their respective regulatory element. ${ }^{22}$ Kasowski et $a^{23}$ have recently reported the presence of considerable inter-individual variation in chromatin states as assessed by histone modifications, and binding of cohesin and CTCF. In particular, they found person-to-person variation in enhancer activity without major changes in gene expression, ${ }^{23}$ confirming the hypothesis of inter-individual variation in the use of redundant enhancers. It is noteworthy that rare variants (allele frequency $<1 \%$ ) seems to explain in part this variation on enhancer use. ${ }^{23}$ Whether TCF7L2 enhancers also show this type of variability is still unknown.

Table 1 shows the 28 elements tested for enhancer activity by Savic et al ${ }^{6,7}$ in the TCF7L2 gene. Positions of the different constructs were obtained by blasting construct primer sequences to the reference human genome sequence version 19. It is worth noting that (a) SNPs associated with T2D risk are either inside or surrounded by elements with enhancer activity, (b) some elements with enhancer activity do not harbor any reported SNP associated with risk of T2D, even

Table 1 Position of the 28 conserved elements in the TCF7L2 gene (chromosome 10) assessed by Savic et al. for enhancer activity

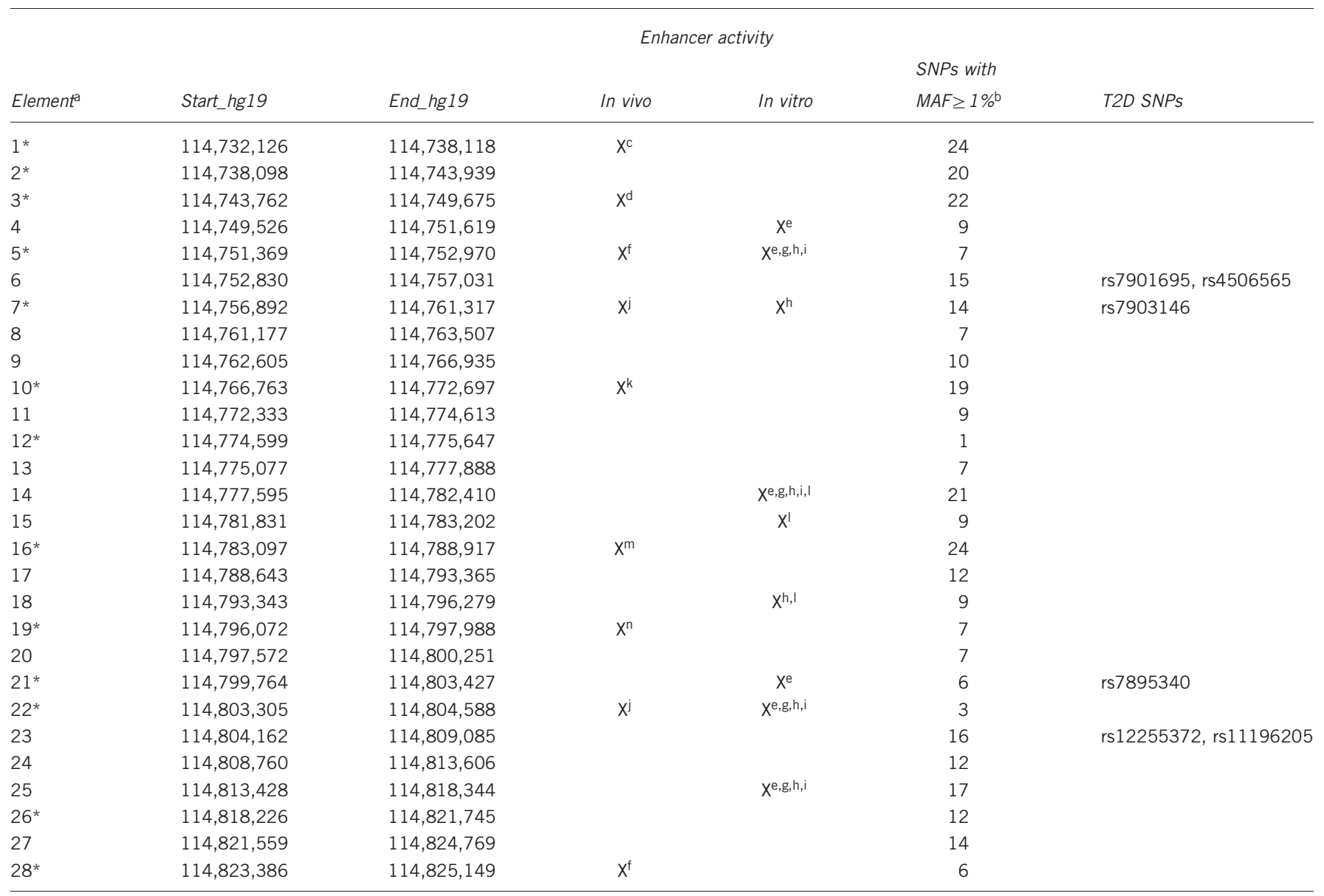

aThe in vivo scan analyzed only 13 elements, those marked by an *; the in vitro scan assessed all the twenty eight conserverd elements.

${ }^{b}$ Number of SNPs with minor allele frequency $\geq 1 \%$ in the combined populations from 1000 genomes project.

cStomach.

dSpinal neurons.

eNeuro-2a cell line.

fBone.

gHCT-116 cell line.

${ }^{\mathrm{h}} \mathrm{C} 2 \mathrm{C} 12$ cell line.

iU20S cell line.

jBrain.

kWalking pads.

IMIN6 cell line.

mLimb vasculature.

nBrain vasculature. 
though they contain polymorphic variants, and (c) elements with enhancer activity harboring T2D risk variants are located on both ends of the 92-kb region. Although the rs12255372 SNP (ie, the one the present letter proposes as an secondary signal) is not located inside any of the elements with enhancer activity reported by Savic et al., ${ }^{6,7}$ chromatin segmentation analysis using the HaploReg software version $2^{24}$ with data from the NIH Roadmap Epigenomics Mapping Consortium (http://www. roadmapepigenomics.org/) shows that rs12255372 is located inside elements with chromatin mark characteristics of active enhancers in 10 different cell types and weak enhancers in 20 different cell types (Table 2). It is noteworthy that some of the tissues represented by these cell lines (eg, the adipose, pancreas, gastric, and brain) are involved in glucose metabolism. The rs7903146 is located inside elements with chromatin mark characteristics of promoter activity in

Table 2 Regulatory chromatin states in different cell lines from the NIH Roadmap Epigenomics Mapping Consortium around SNPs rs7903146 and rs12255372 in the TCF7L2 gene

Chromatin states in different cell lines

\begin{tabular}{|c|c|c|c|c|}
\hline SNP & $\begin{array}{c}\text { Promoter } \\
\text { activity }\end{array}$ & $\begin{array}{c}\text { Weak promoter } \\
\text { activity }\end{array}$ & Enhancer activity & $\begin{array}{l}\text { Weak enhancer } \\
\text { activity }\end{array}$ \\
\hline
\end{tabular}

\begin{tabular}{llll}
\hline rs7903146 ADI.MSC PANC.I & ADI.NUC & BN.HM150 \\
& HD.CD184EC & BR.MYO
\end{tabular}

$\begin{array}{ll}\text { HD.CD184EC } & \text { BR.MYO } \\ \text { HUES48 } & \text { COL.SMUS }\end{array}$

LNG.FE

ST.MUC

rs12255372 ADI.NUC ADI.MSC

BN.AG BM.MSC

BN.HM150 BN.AC

BN.SN BN.FE2

HD.CD56MESC BR.MYO

H1.DMSC DUO.SMUS

MSC.ADIPC ES.I3

NCC.COR2 GAS

PFF.1 HUES6

PFF.2 HUES64

H1.BMP4DT

H1.DNP

$\mathrm{H} 9$

IPS.DF6

IPS.DF19

IPS. 15

IPS. 18

IPS.20

NCC.GED2

PANC

Abbreviations: ADI.MSC, adipose-derived mesenchymal stem cell; ADI.NUC, adipose nuclei; BM.MSC, bone marrow-derived mesenchymal stem cell; BN.AC, brain anterior caudate; BN.AG, brain angular gyrus; BN.FE2, fetal brain donor HuFNSCO2; BN.HM150, brain hippocampus middle donor 150; BN.SN, brain substantia nigra; BR.MYO, breast myoepithelial cells; COL.SMUS, colon smooth muscle; DUO.SMUS, duodenum smooth muscle; ES.I3, embryonic stem cell-I3; GAS, gastric; HD.CD184EC, hESC-derived CD184 + endoderm cultured cells; HD.CD56MES, ,ESC-d; D.CD5GMESC, , gSC-di; HD.CD184EC, hESC-derived CD184+ end PDT, H1 BMP4-deric stem cells-48; HUES64, human embryonic stem cells-64; H1.BMP4DT, H1 BMP4-derived trophoblast-cultured cells; H1.DMSC, H1-derived
mesenchymal stem cells; H1.DNP, H1-derived neuronal progenitor-cultured cells; H9, H9 cell line; IPS.DF6, induced pluripotent stem cells DF 6.9; IPS.DF19, induced pluripotent stem cells DF 19.11; IPS.15, induced pluripotent stem cells 15b; IPS.18, induced pluripotent stem cells 18; IPS.20, induced pluripotent stem cells 20b; LNG.FE, fetal lung; MSC.ADIPC, mesenchymal stem cell-derived adypocyte cultured cells; NCC.COR2, neurosphere-cultured cells cortex-derived donor HUFNSCO2; NCC.GED2, neurosphere-cultured cells ganglionic eminence-derived donor HuFNSC02; PANC, pancreas; PANC.I, pancreatic islets; PFF.1, penis foreskin fibroblast primary cells donor skin01; PFF.2, penis foreskin fibroblast primary cells donor skin02; ST.MUC, stomach mucosa. one cell line, weak promoter activity in one cell line, enhancer activity in three cell lines, and weak enhancer activity in five cell lines (Table 2). These results suggest that both rs7903146 and rs12255372 may affect risk of T2D through enhancer-mediated mechanisms. On the basis of the proposed hypothesis of inter-individual variation in the use of redundant enhancers, rs7903146 would show the strongest association with T2D in most of the studied populations because its respective enhancer would be the most frequently used. However, it is possible that in populations with a different usage of redundant enhancers other TCF7L2 variants may also affect risk of T2D. Presence of additional functional SNPs is suggested by the recent functional analysis of TCF7L2 polymorphisms by Pang et al. who found that five SNPs including rs7903146 showed allele-specific binding patterns in electrophoretic mobility shift assays. These results need in vivo confirmation as in vitro assays do not give a complete picture in how the complex chromatin structure inside cells affects binding of transcription factors. For example, Chakrabarti et $a^{26}$ reported that in vitro immunoprecipitation assays overestimate the number of binding sites of the transcription factor $\mathrm{Pdx} 1$ in both $\beta$-cells and non $\beta$-cells as measured by chromatin immunoprecipitation. Nevertheless three of the TCF7L2 SNPs assessed by Pang et al ${ }^{25}$ had allele-specific luciferase activity in both Huh7 (hepatocarcinoma) and WiDr (colorectal adenocarcinoma) cell lines (rs4132670), or in Huh7 cell line only (rs4506565, and rs12255372), ${ }^{25}$ suggesting that these SNPs may have in vivo functional relevance. Although results from these two nonpancreatic tumor-derived cell lines must be interpreted with caution, they stress the need to assess TCF7L2 SNP function in a variety of organs other than pancreas, as it has been shown that TCF7L2 regulates glucose homeostasis through its action in a variety of nonpancreatic tissues such as the liver, ${ }^{27,28}$ gut, ${ }^{29}$ and brain. ${ }^{30}$

\section{Testing the hypothesis of alternative use redundant enhancers} in the TCF7L2 gene

Testing the hypothesis of person-to-person variation in the use of redundant enhancers in the TCF7L2 gene requires the ability of distinguishing active enhancers from poised/inactive enhancers. Recent work has shown that active enhancers are bi-directionally transcribed resulting in short $(<2 \mathrm{~kb})$ noncoding RNAs (enhancer RNAs or eRNAs). ${ }^{31}$ Zhu et al ${ }^{32}$ reported that expression levels of eRNAs, and therefore enhancer activity, can be reliably predicted by assessing $\mathrm{H} 3 \mathrm{~K} 27 \mathrm{ac}, \mathrm{H} 3 \mathrm{~K} 27 \mathrm{me} 3, \mathrm{H} 3 \mathrm{~K} 79 \mathrm{~m} 1$, and $\mathrm{H} 3 \mathrm{~K} 9 \mathrm{ac}$ histone modifications (area under the curve $(\mathrm{AUC})=0.94$, and Matthew's correlation coefficient $(\mathrm{MCC})=0.71)$, and the predictive model seems to be cell-type independent. These observations offer an approach to measure enhancer activity and to test the hypothesis that active enhancers vary from person to person. Preliminary chromatin segmentation analysis of the 92-kb enhancer-rich region of the TCF7L2 gene using available data from different cells/tissues with more than one donor from the NIH Roadmap Epigenomics Mapping Consortium (http://www.roadmapepigenomics.org/) shows variability on chromatin states from tissue-to-tissue and from personto-person for the same tissue (Supplementary Figure 1). It is noteworthy that the rs 12255372 SNP (ie, the one proposed as a secondary signal) is located inside regions with weak enhancer activity in some of the tissues. A crucial next step should be the measurement of enhancer activity by assessing histone modifications in the whole range of tissues where TCF7L2 is expressed. This assessment must move from the traditional 2-dimension approach enhancer $\mathrm{x}$ tissue to a more comprehensive 3-dimension scenario subject $\mathrm{x}$ enhancer $\mathrm{x}$ tissue (ie, to allow for inter-individual variation in the use of redundant enhancers). 


\section{Summary}

TCF7L2 is the gene with the strongest association with T2D, and it is generally accepted that the rs7903146 SNP is the single causal variant in this locus. ${ }^{8-10}$ The present letter discussed published data from European and nonEuropean populations and postulated that an independent secondary signal tagged by the rs12255372 SNP is also present in the TCF7L2 gene. The author also discussed recent results that show the presence of multiple enhancers in the TCF7L2 gene, ${ }^{6,7}$ and advanced the hypothesis that these enhancers are redundant and there is person-to-person variability in their use. This hypothesis may explain in part why the rs12255372 shows a weaker association with T2D compared with rs7903146; namely rs12255372 would be located inside elements with weak enhancer activity and with less frequency of use. Preliminary analysis of available data from the NIH Roadmap Epigenomics Mapping Consortium (http://www. roadmapepigenomics.org/) seems to bring support to this hypothesis. A comprehensive assessment of enhancer functional redundancy in all tissues where TCF7L2 is expressed may clarify the interplay between genetic variants and chromatin modifications, and how they jointly affect the development of T2D.

\section{CONFLICT OF INTEREST}

The author declares no conflict of interest.

\section{ACKNOWLEDGEMENTS}

The author would like to thank Dr Marcelo Nobrega for providing primer sequences of the constructs that were used by Savic et $a l^{6,7}$ in the scanning for elements with enhancer activity in TCF7L2. This work was supported by grants R01MD007015 from the National Institute on Minority Health and Health Disparities, R01CA058420 from the National Cancer Institute, and 11SDG7390014 from the American Heart Association.

\section{DISCLAIMER}

The content is solely the responsibility of the author and does not necessarily represent the official views of the National Institute on Minority Health and Health Disparities, the National Cancer Institute, the National Institutes of Health, or the American Heart Association.

\section{Edward A Ruiz-Narváez ${ }^{*, 1,2}$}

${ }^{1}$ Slone Epidemiology Center at Boston University, Boston, MA, USA; ${ }^{2}$ Department of Epidemiology, Boston University School of Public Health, Boston, MA, USA E-mail: eruiznar@bu.edu

1 Morris AP, Voight BF, Teslovich TM et al: Large-scale association analysis provides insights into the genetic architecture and pathophysiology of type 2 diabetes. Nat Genet 2012; 44: 981-990.

2 Saxena R, Elbers CC, Guo Y et al: Large-scale gene-centric meta-analysis across 39 studies identifies type 2 diabetes loci. Am J Hum Genet 2012; 90: 410-425.

3 Gaulton KJ, Nammo T, Pasquali $L$ et al: A map of open chromatin in human pancreatic islets. Nat Genet 2010; 42: 255-259.

4 Stitzel ML, Sethupathy P, Pearson DS et al: Global epigenomic analysis of primary human pancreatic islets provides insights into type 2 diabetes susceptibility loci. Cell Metab 2010; 12: 443-455
5 Lyssenko V, Lupi R, Marchetti P et al: Mechanisms by which common variants in the TCF7L2 gene increase risk of type 2 diabetes. J Clin Invest 2007; 117: 2155-2163.

6 Savic D, Bell GI, Nobrega MA: An in vivo cis-regulatory screen at the type 2 diabetes associated TCF7L2 locus identifies multiple tissue-specific enhancers. PLoS One 2012; 7: e36501.

7 Savic D, Park SY, Bailey KA, Bell GI, Nobrega MA: In vitro scan for enhancers at the TCF7L2 locus. Diabetologia 2013; 56: 121-125.

8 Helgason A, Palsson S, Thorleifsson G et al: Refining the impact of TCF7L2 gene variants on type 2 diabetes and adaptive evolution. Nat Genet 2007; 39 218-225.

9 Palmer ND, Hester JM, An SS et al: Resequencing and analysis of variation in the TCF7L2 gene in African Americans suggests that SNP rs7903146 is the causal diabetes susceptibility variant. Diabetes $2011 ; 60$ : 662-668.

10 Maller JB, McVean G, Byrnes J et al: Bayesian refinement of association signals for 14 loci in 3 common diseases. Nat Genet 2012; 44: 1294-1301.

11 Grant SF, Thorleifsson G, Reynisdottir I et al: Variant of transcription factor 7-like 2 (TCF7L2) gene confers risk of type 2 diabetes. Nat Genet 2006; 38: 320-323.

12 Sale MM, Smith SG, Mychaleckyj JC et al: Variants of the transcription factor 7-like 2 (TCF7L2) gene are associated with type 2 diabetes in an African-American population enriched for nephropathy. Diabetes 2007; 56: 2638-2642.

13 Parra EJ, Cameron E, Simmonds L et al: Association of TCF7L2 polymorphisms with type 2 diabetes in Mexico City. Clin Genet 2007; 71: 359-366.

14 Mattei J, Qi Q, Hu FB, Sacks FM, Qi L: TCF7L2 genetic variants modulate the effect of dietary fat intake on changes in body composition during a weight-loss intervention. Am J Clin Nutr 2012; 96: 1129-1136.

$15 \mathrm{Hu}$ G, Codina M, Fisher S: Multiple enhancers associated with ACAN suggest highly redundant transcriptional regulation in cartilage. Matrix Biol 2012; 31: 328-337.

16 Degenhardt KR, Milewski RC, Padmanabhan A et al: Distinct enhancers at the Pax3 locus can function redundantly to regulate neural tube and neural crest expressions. Dev Biol 2010; 339: 519-527.

17 Bernstein BE, Birney E, Dunham I, Green ED, Gunter C, Snyder M: An integrated encyclopedia of DNA elements in the human genome. Nature 2012; 489: 57-74.

18 Demura M, Bulun SE: CpG dinucleotide methylation of the CYP19 I.3/II promote modulates cAMP-stimulated aromatase activity. Mol Cell Endocrinol 2008; 283: 127-132.

19 Demura M, Demura Y, Ameshima S et al: Changes in aromatase (CYP19) gene promoter usage in non-small cell lung cancer. Lung Cancer 2011; 73: 289-293.

20 Ishikawa H, Reierstad S, Demura M et al: High aromatase expression in uterine leiomyoma tissues of African-American women. J Clin Endocrinol Metab 2009; 94: 1752-1756.

21 Ruiz-Narvaez EA: What is a functional locus? Understanding the genetic basis of complex phenotypic traits. Med Hypotheses 2011; 76: 638-642.

22 Ruiz-Narvaez EA: Use of alternative promoters may hide genetic effects on phenotypic traits. J Hum Genet 2013; 58: 47-50.

23 Kasowski M, Kyriazopoulou-Panagiotopoulou S, Grubert F et al: Extensive variation in chromatin states across humans. Science 2013; 342: 750-752.

24 Ward LD, Kellis M: HaploReg: a resource for exploring chromatin states, conservation, and regulatory motif alterations within sets of genetically linked variants. Nucleic Acids Res 2012; 40: D930-D934.

25 Pang DX, Smith AJ, Humphries SE: Functional analysis of TCF7L2 genetic variants associated with type 2 diabetes. Nutr Metab Cardiovasc Dis 2013; 23: 550-556.

26 Chakrabarti SK, James JC, Mirmira RG: Quantitative assessment of gene targeting in vitro and in vivo by the pancreatic transcription factor, Pdx1. Importance of chromatin structure in directing promoter binding. J Biol Chem 2002; 277: $13286-13293$.

27 Boj SF, van Es JH, Huch M et al: Diabetes risk gene and Wnt effector Tcf7I2/TCF4 controls hepatic response to perinatal and adult metabolic demand. Cell 2012; 151 : 1595-1607.

28 Oh KJ, Park J, Kim SS, Oh H, Choi CS, Koo SH: TCF7L2 modulates glucose homeostasis by regulating $\mathrm{CREB}$ - and FoxO1-dependent transcriptional pathway in the liver. PLoS Genet 2012; 8: e1002986.

29 Yi F, Brubaker PL, Jin T: TCF-4 mediates cell type-specific regulation of proglucagon gene expression by beta-catenin and glycogen synthase kinase-3beta. J Biol Chem 2005; 280: 1457-1464.

30 Shao W, Wang D, Chiang YT et al: The Wnt signaling pathway effector TCF7L2 controls gut and brain proglucagon gene expression and glucose homeostasis. Diabetes 2013; 62: 789-800.

$31 \mathrm{Kim}$ TK, Hemberg M, Gray JM et al: Widespread transcription at neuronal activityregulated enhancers. Nature 2010; 465: 182-187.

32 Zhu Y, Sun L, Chen Z, Whitaker JW, Wang T, Wang W: Predicting enhancer transcription and activity from chromatin modifications. Nucleic Acids Res 2013; 41: $10032-10043$

Supplementary Information accompanies this paper on European Journal of Human Genetics website (http://www.nature.com/ejhg) 